Original Research Paper

\title{
Use of Maple to Analytically Solve the Equations of an Electrical Circuit Containing a Resistor, Diodes and Voltage Generator
}

\author{
${ }^{1,4}$ M'hamed El Aydi, ${ }^{2}$ Mohammed Barkatou, ${ }^{1}$ Rachid Bendaoud, ${ }^{3}$ Mohammed Sbaa and ${ }^{1}$ Said Bounouar \\ ${ }^{1}$ Department of Physics, Faculty of Sciences, U.C.D, EL Jadida, Morocco \\ ${ }^{2}$ Department of Mathematics, Faculty of Sciences, UCD, EL Jadida, Morocco \\ ${ }^{3}$ Departement of Mathematics, Faculty of Sciences and Technology, Settat, Morocco \\ ${ }^{4}$ Department of Mathematics, Regional Centre of the Jobs of Education and Training, El Jadida, Morocco
}

\begin{abstract}
Article history
Received: 26-09-2020

Revised: 30-11-2020

Accepted: 01-12-2020

Corresponding Author:

M'hamed El Aydi

Department of Physics, Faculty of Sciences, U.C.D, EL Jadida, Morocco

Email: elaydi58@gmail.com
\end{abstract}

\begin{abstract}
The role of technology and the use of software in the educational process are growing in recent times. The use of software is essential especially if the analytical method available is too complicated for the students. In this study, we used the Maple software to deal with two physics problems, in the first problem we consider an electrical circuit containing a resistor and two diodes powered by a sinusoidal voltage generator and in the second problem we consider an electrical circuit containing a resistor and a diode powered by a saw tooth voltage generator. For each problem we use Maple software to determine the exact analytical solutions for the current flowing in the different branches of the electronic circuit, we derive analytical expressions for the terminal voltages of all the elements of the circuit, we calculate the dynamic resistances diodes of the circuit and we animate graphic representations to study the influence of certain parameters on the current and the voltages at the terminals of all the elements of the circuit. The analytical solutions proposed are all expressed as functions of the Lambert $\mathrm{W}$ function.
\end{abstract}

Keywords: The PN Junction Diode, Dynamical Resistances the Diodes, Lambert W Function, Maple Software, Saw Tooth Excitation Voltage

\section{Introduction}

Maple is a proprietary computer algebra software allowing to manipulate mathematical expressions symbolically and thus to make exact calculations. Maple is a computer environment for advanced mathematics including tools for algebra, referential equations, mathematical analysis, discrete mathematics, graphical and numerical calculation, etc.

The transcendent equation of current intensity through a diode driven by a voltage source through a serial resistor is usually solved by accepting approximations. Fjeldly et al. (1991) exploited an approximate analytical resolution technique combined a test function with a series of expansion. This method leads to a precise solution without requiring a lot of computing time.

The authors (Pimbley et al., 1992) used Newton's method provides an accurate solution for negative values of normalized tension, but the precision of the solution is less acceptable for very large values of the normalized tension. Moreover, this method induced a lot of computing time.

In the work published by (Banwell and Jayakumar, 2000), the authors used the LambertW function to express the exact analytical solution for the normalized form of the generalized diode equation. The researcher in (Vargas-Drechsler, 2005) derived the same exact analytical solution using the computer algebra software Maple (Eberhart, 2009).

In this application worksheet, we consider two problems:

- In the first problem, we consider an electrical circuit containing a resistor and two diodes powered by a sinusoidal voltage generator "Fig. 1", we use Maple software to determine exact 
analytical solutions for the current flows through the different branches of the electronic circuit. Then we derive analytical expressions for the voltages at the terminals of all the elements of the circuit and we represent them graphically using the Maple software. Finally, we calculate the dynamical resistances the diodes in the circuit. The proposed analytical solutions are all expressed as functions of the Lambert $\mathrm{W}$ function

- In the second problem: Maple is used to determine exact analytical solution for the current flows through the Electrical circuit containing a resistor and diode powered by a saw tooth voltage generator presented in Fig. 2 and to study the influence of four parameters involved

\section{First Problem}

Exact analytical solution in electronic circuit containing a resistor and two diodes powered by a sinusoidal voltage generator (Fig. 1).

\section{Materials and Methods}

In this section, we introduce the used materials an methods: LambertW function (Dence, 2013), Kirchhoff's current law, Kirchhoff's voltage law, saturation current, dynamical resistance, Maple software.

\section{LambertW Function}

The function Lambert $W$ is the reciprocal of the function $f(x)=x e^{x}$. Since the equation $x \cdot \exp (x)=y$ has an infinite number of solutions for a non-zero complex value. The function Lambert $W$ admits an infinite number of branches in the complex plane and only two of these branches are real:

i) The first branch, also called the main branch, vanishes at 0 , it is noted LambertW0 and is defined from $[-1 / e,+\infty]$ verses $[-1,+\infty]$

ii) The second branch is noted LambertW-1 and is defined from $[-1 / e, 0]$ verses $[-\infty,-1]$

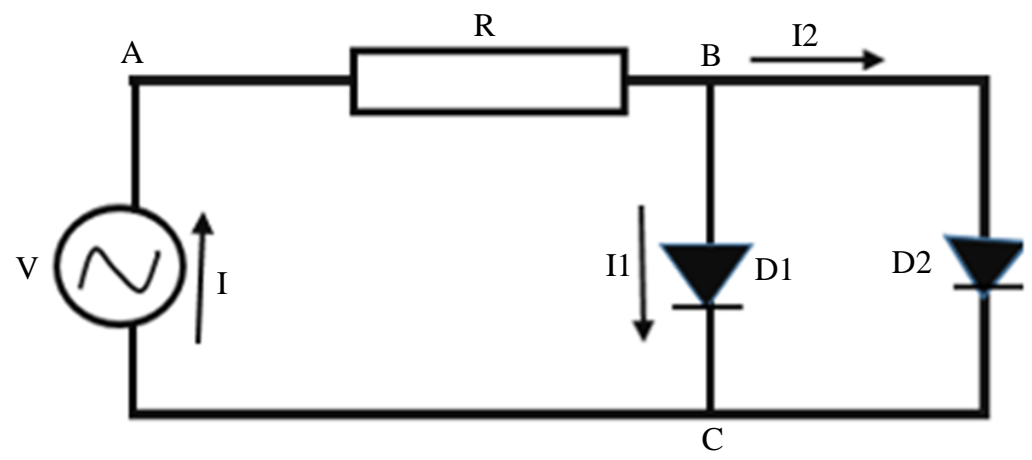

Fig. 1: Electronic circuit containing a resistor and two diodes

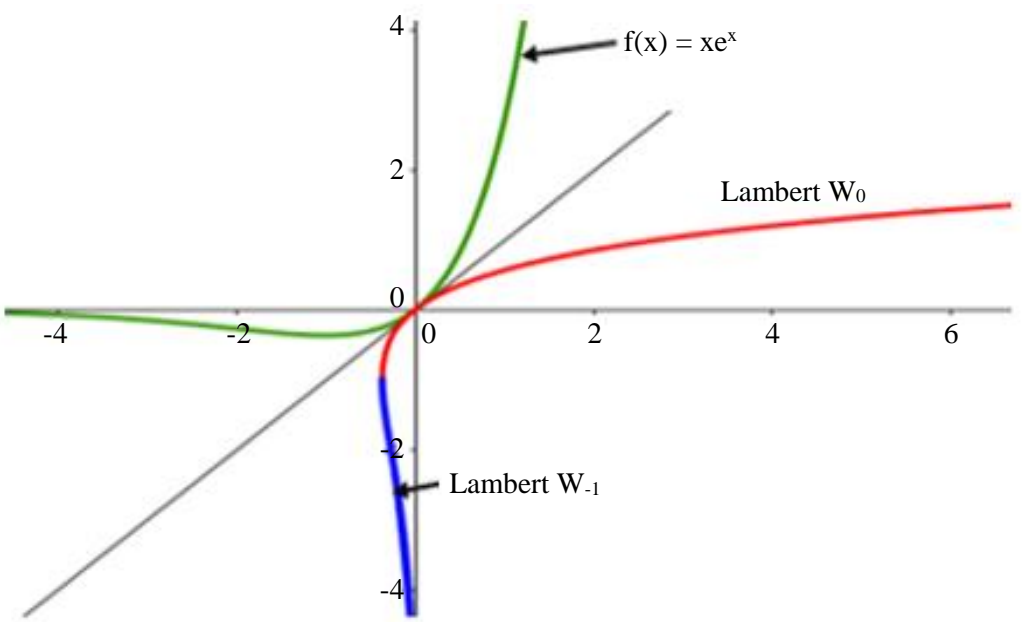

Fig. 2: Graph of Lambert W function 


\section{Kirchhoff's Current Law}

Kirchhoff's Current Law (KCL) states that the algebraic sum of currents entering a node (or a closed boundary) is zero.

Kirchhoff's Voltage Law (KVL) states that the algebraic sum of all voltages around a closed path (or loop is zero.).

The current through the diode is:

- $\quad I=I s\left(\exp (q V /(\text { eta.kB.T })-1)^{1}\right.$

- Dynamic resistance ${ }^{2} r$ is, $r=d V / d I$

- Ohm's law ${ }^{3}$ is: $V=R I$

- $\quad$ Maple problem solving handbook (Eberhart, 2009)

Modeling the Problem using Maple Software

\section{Nomenclature}

$R: \quad$ Resistance

D1: Diode, $I s 1$ its saturation current

D2: Diode, $I s 2$ its saturation current

$I=I R$ : Current through the resistance

I1: $\quad$ Current through the diode $D 1$

I2: $\quad$ Current through the diode D2

$q$ : $\quad$ Electron charge

eta: Ideality factor of the diodes

$k B$ : Boltzmann constant

$T: \quad$ The absolute temperature

$V: \quad$ Sinusoidal voltage source

$V A B:$ Voltage across the resistance

$V B C$ : Voltage across the diodes

$R d 1: \quad$ Dynamical resistance of the diode $D 1$

$R d 2: \quad$ Dynamical resistance of the diode $D 2$

$P: \quad$ Period of V $a=\frac{q}{\text { eta.kB.T }}$

Note that the calculations, Equation solving, Graphic representations and Animations will be done using the Maple software.

\section{The Current Flowing Through the Resistor}

>restart:

Using Kirchhoff’s current law:

$>$ eql $:=I R=I 1+I 2$

eql $:=I R=I 1+I 2$

Using Ohm's law:

$>e q 2:=V A B=R \cdot I R$

eq2 $:=V A B=R I R$

Using the current through the diode D1:

${ }^{1}$ https://www.pveducation.org/pvcdrom/pn-junctions/diode-equation ${ }^{2}$ http://fourier.eng.hmc.edu/e84/lectures/ch4/node2.html

${ }^{3}$ https://en.wikipedia.org/wiki/Ohm\%27s_law $>e q 3:=I 1=I s 1 \cdot(\exp (a \cdot V B C)-1)$

$e q 3:=I 1=I s 1\left(e^{a V B C}-1\right)$

Using the current through the diode D2:

$>e q 4:=I 2=I s 2 \cdot(\exp (a \cdot V B C)-1)$

$e q 4:=I 2=I s 2\left(e^{a V B C}-1\right)$

Using Kirchhoff's voltage law:

$>e q 5:=V=V A B+V B C$

$e q 5:=V=V A B+V B C$

$>$ eq6 :=VBC= solve $(e q 5, V B C)$;

eq6 $:=V B C=-V A B+V$

$>e q 7:=\operatorname{subs}(e q 2, e q 6)$

$e q 7:=V B C=-I R R+V$

$>e q 8:=\operatorname{subs}(e q 7, e q 3)$

$e q 8:=I 1=I s 1\left(e^{a(-I R R+V)}-1\right)$

$>$ eq $9:=\operatorname{subs}(e q 7, e q 4)$

$e q 9:=I 2=I s 2\left(e^{a(-I R R+V)}-1\right)$

$>$ eq $10:=\operatorname{subs}(e q 9, e q 1)$

$e q 10:=I R=I 1=I s 2\left(e^{a(-I R R+V)}-1\right)$

$>e q 11:=\operatorname{subs}(e q 8, e q 10)$;

$e q 11:=I R=I s 1\left(e^{a(-I R R+V)}-1\right)+I s 2\left(e^{a(-I R R+V)}-1\right)$

$>I R:=$ solve $(e q 11, I R)$;

$\mathrm{IR}:=\frac{I s 1 R a+I s 2 R a-\text { LambertW }\left(R a(I s 1+I s 2) e^{I s 1 R a+I s 2 R a+V a}\right)}{R a}$

The Current Flow $i$ Through a PN Junction is Related to the Voltage $v$ at its Terminals by the Relation

$>e q 12:=i=I s^{*}\left(\exp \left(a^{*} v\right)-1\right)$

$e q 12:=i=I s\left(e^{a v}-1\right)$

$>v:=\operatorname{solve}(e q 12, v)$

$v:=\frac{\ln \left(\frac{I s+i}{I s}\right)}{a}$

Dynamical Resistance of the Diode D

$>R d:=\operatorname{diff}(v, i)$

$R d:=\frac{1}{(I s+i) a}$

Thus:

$R d 1:=\frac{1}{(I s 1+I 1) a}$ and $R d 2:=\frac{1}{(I s 2+I 2) a}$

Graphs of Current Flows and Voltages of the Circuit against Time

$>$ restart:

$>$ withplots: 
$>V:=t \rightarrow V m^{*} \sin \left(\right.$ omege $\left.^{*} t\right) ; a:=\frac{q}{\text { eta.kB.T}}$

$V:=t \rightarrow V m \sin (\omega t)$

$a:=\frac{q}{\eta \cdot k B \cdot T}$

$>\quad I R:=\quad t \rightarrow \quad \frac{1}{a \cdot R} \quad \cdot$ LambertW( $a \cdot(R \cdot(I s 1 \quad+$

$I s 2) \cdot \exp (a \cdot(R \cdot(I s 1+I s 2)+V(t))))-(I s 1+I s 2)$

$\operatorname{IR}:=\mathrm{t} \rightarrow \frac{\text { LambertW }\left(a R(I s 1+I s 2) e^{a(R(I s 1+I s 2)+V(t))}\right)}{a R}-I s 1-I s 2$

$>V A B:=t \rightarrow R \cdot I R(t)$

$V A B:=t \rightarrow R I R(t)$

$>V B C:=t \rightarrow V(t)-R \cdot I R(t)$

$V B C:=t \rightarrow V(t)-R I R(t)$

The numerical values used are such that:

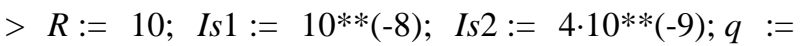
$1.60219 * 10 * *(-19)$;

$R:=10$

$I s 1:=\frac{1}{100000000}$

Is $2:=\frac{1}{250000000}$

$q:=1.60219000010^{-19}$

$>$ eta $:=1.8 ; k B:=1.38062 * 10 * *(-23) ; \quad T:=300$;

omega $:=2 * \mathrm{Pi}^{*} 60 ; \mathrm{Vm}:=3$;

$\eta:=1.8$

$k B:=1.38062000010^{-23}$

$T:=300$ $\omega:=120 \pi$

$V m:=3$

$>I 1:=t \rightarrow I s 1 \cdot(\exp (a \cdot V B C(t))-1):$

$I 2:=t \rightarrow I s 2 \cdot(\exp (a \cdot V B C(t))-1)$ :

$>R d 1:=\frac{1}{(I s 1+I 1(t)) \cdot a}: R d 2:=\frac{1}{(I s 2+I 2(t)) \cdot a}:$

Graphical Representation of the Voltage Across the Resistance and Voltage Across the Diodes

$>\operatorname{plot}\left([V A B(t), V B C(\mathrm{t})], t=0 . . \frac{2 * P i}{\text { omega }}\right.$, color $=[$ black, blue]);

Graphical Representation of the Current Flowing through the Resistor, the Diode1, the Diode2 and $I 1(t)+I 2(t)$

$>\operatorname{plot}\left([I R(t), I 1(t), I 2(t), I 1(t)+I 2(t)], t=0 . . \frac{2^{*} P i}{\text { omega }}\right.$,

color $=[$ black, red, blue, brown $])$;

Graphical Representation of II(t) and I2(t)

$>\operatorname{plot}\left([I 1(t), I 2(t)], t=\frac{P i}{\text { omega }} . . \frac{2 * P i}{\text { omega }}\right.$, color $=[$ red, blue $\left.]\right) ;$

Graphical Representation Dynamic Resistances of Diodes D1 and D2

$>$ plot $\left([R d 1(t), R d 2(t)], \mathrm{t}=0 . . \frac{4^{*} P i}{\text { omega }}\right.$, color $=[$ black, blue]);

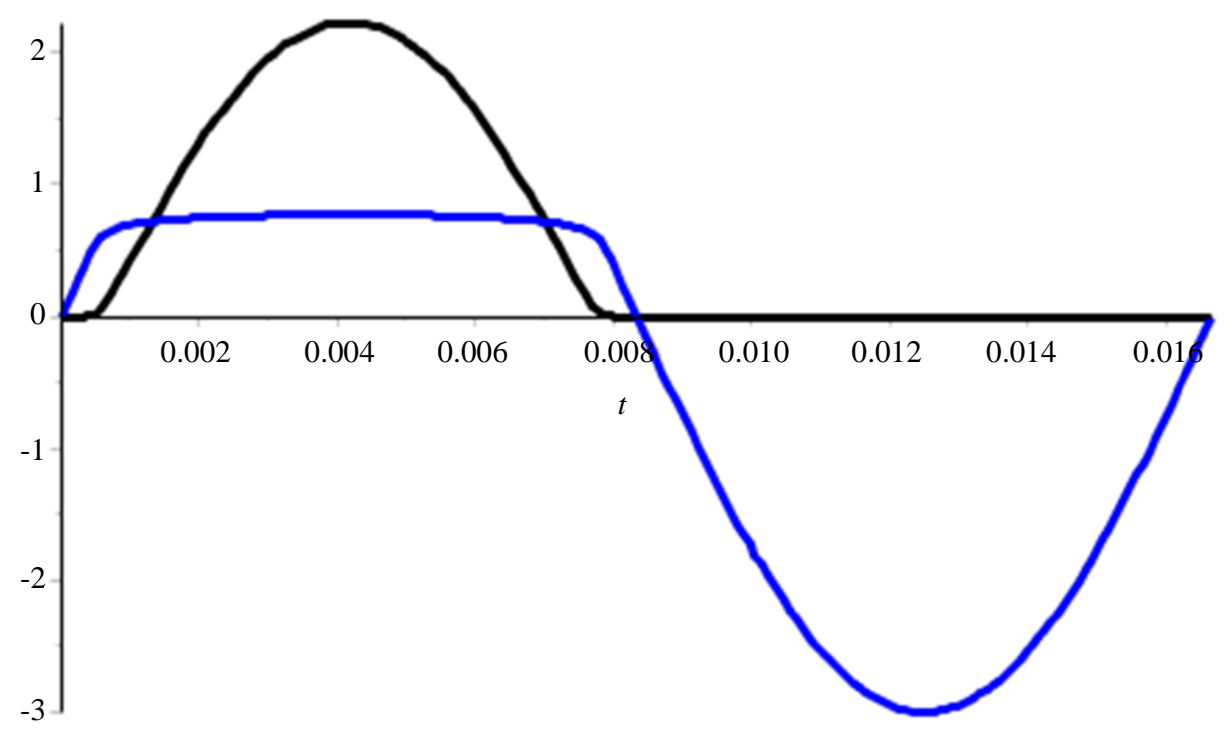

Fig. 3: Graphical representation of the $\mathrm{VAB}$ and $\mathrm{VBC}$ in the interval of time $[0, \mathrm{P}]$ 


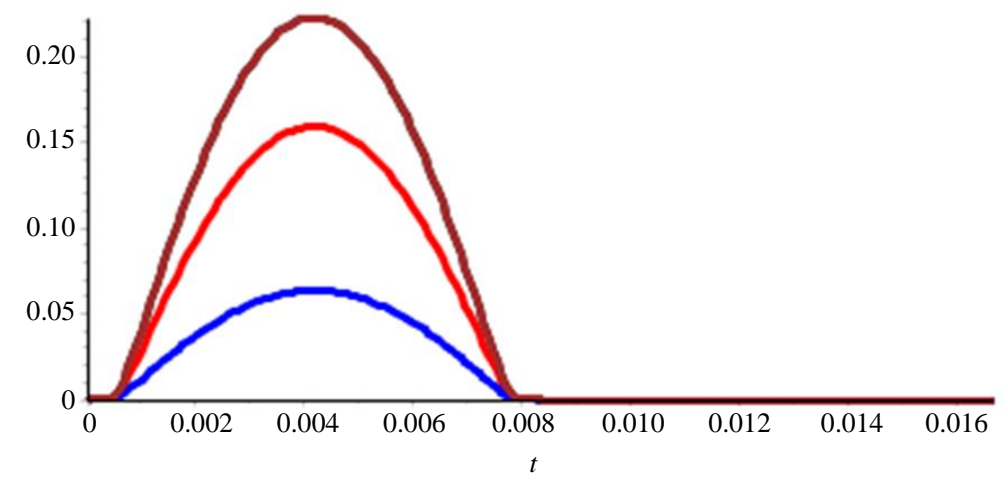

Fig. 4: Graphical representation of $I R(t), I 1(t), I 2(t)$ and $I 1(t)+I 2(t)$ in the interval of time $[0, \mathrm{P}]$

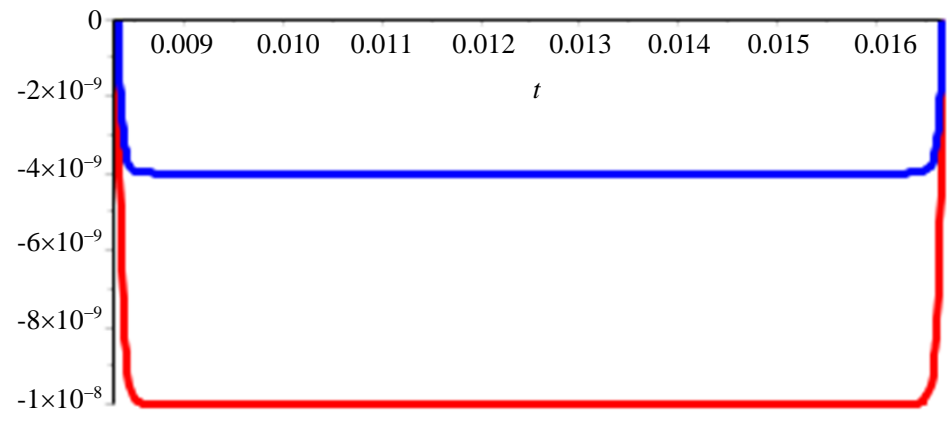

Fig. 5: Graphical representation of $\mathrm{I} 1(\mathrm{t})$ and $\mathrm{I} 2(\mathrm{t})$ in the interval of time $[\mathrm{P} / 2, \mathrm{P}]$

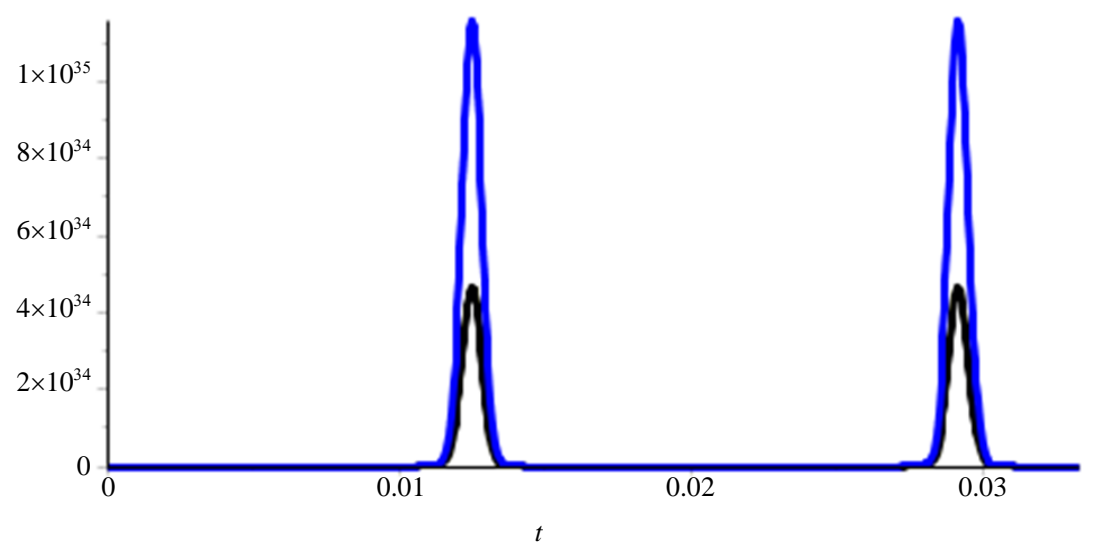

Fig. 6: Graphical representation $R d 1$ and $R d 2$ in the interval of time [0,2P]

\section{Second Problem}

We considered the electrical circuit containing a resistor and a diode powered by a saw tooth voltage generator Fig. 7, we want to determine the analytical expressions for the voltages at the terminals of all elements in the circuit and we are interested in studying the influence of the saturation current, the temperature and the ideality factor.

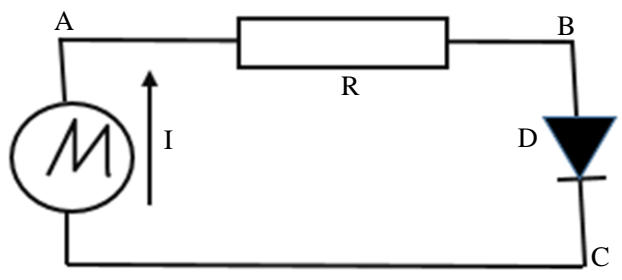

Fig. 7: Electrical circuit containing a resistor and diode powered by a saw tooth voltage generator 


\section{Modeling the Problem using Maple Software}

\section{Nomenclature}

\section{$R: \quad$ Resistance}

$D$ : Diode, Is its saturation current

$I R$ : Current through the resistance equal to the current through the diode

$q$ : Electron charge

eta: Ideality factor of the diode

$k B$ : Boltzmann constant

$T: \quad$ Is the absolute temperature

$V$ : Voltage source

$V A B$ : Voltage across the resistance

$V B C$ : Voltage across the diode

$R d$ : Dynamical résistance of the diode $a=\frac{q}{\text { eta.kB.T }}$

The calculations, Equation solving, Graphic representations and Animations will be done using the Maple software.

The current flowing through the resistor:

$>$ restart:

Using Ohm's law:

$>e q 1:=V A B=R \cdot I R$

$e q 1:=V A B=R I R$

Using the current through the diode D:

$>e q 2:=I R=I s \cdot(\exp (a \cdot V B C)-1)$

$e q 2:=I R=I s\left(\mathrm{e}^{a V B C}-1\right)$

Using Kirchhoff's voltage law:

$>$ eq $3:=\mathrm{V}$

$e q 3:=V=V A B+V B C$

$e q 3:=V=V A B+V B C$

$>e q 4:=\operatorname{subs}(e q 1, e q 3)$;

$e q 4:=V=I R R+V B C$

$>$ eq $5:=V B C=$ solve $(e q 4, V B C)$;

$e q 5:=V B C=-I R R+V$

$>e q 6:=\operatorname{subs}(e q 5, e q 2)$

$e q 6:=I R=I s\left(e^{a(-I R R+V)}-1\right)$

$>e q 7:=i=I s \cdot(\exp (a \cdot v)-1)$

$e q 7:=i=I s\left(\mathrm{e}^{a v}-1\right)$

$>v:=\operatorname{solve}(e q 7, v)$;

$v:=\frac{\ln \left(\frac{I s+i}{I s}\right)}{a}$

$>R d:=\operatorname{diff}(v, i)$

$R d:=\frac{1}{(I s+i) a}$

$>I R:=\operatorname{solve}(e q 6, I R)$
$I R:=-\frac{I s R a-\text { LambertW }\left(\text { Is Ra } e^{I s R a+V a}\right)}{R a}$

$>I R:=\operatorname{simplify}(I R)$

$I R:=-\frac{I s R a-\text { LambertW }\left(I s R a e^{a(I s R+V)}\right)}{R a}$

$>I R:=\operatorname{expend}(I R)$

$I R:=-I s+\frac{\text { LambertW }\left(I s R a e^{a(I S R+V)}\right)}{R a}$

So the current flowing through the resistor is:

$I R:=-I s+\frac{\text { LambertW }\left(I s R a e^{a(I s R+V)}\right)}{R a}$

In this problem, we consider a sawtooth generator circuit, the mathematical expression of its voltage is given by:

$$
V:=t \rightarrow V m\left(t-\frac{1}{2} P\right) ; t=0 . P ; \text { Pis the period }(V m=0 . .580)
$$

Graphs of Current Flows and Voltages in the circuit as a function of time:

> restart: with(plots):

$>V:=t \rightarrow V m^{*}\left(t-\frac{P}{2}\right)$;

$V:=t \rightarrow V m\left(t-\frac{1}{2} P\right)$

$>a:=\frac{q}{\text { eta.kB.T}}$;

$a:=\frac{q}{\eta \cdot k B \cdot T}$

$>\quad A:=\quad t \rightarrow\left(\frac{1}{a^{*} R}\right) *\left(\right.$ LambertW $\left(a^{*} R^{*} I s^{*} \exp \left(a^{*}(V(t)+\right.\right.$

$\left.\left.\left.R^{*}(s)\right)\right)\right)-I s$

$\mathrm{A}:=t \rightarrow \frac{\text { Lambert } W\left(a R I s e^{a(V(t)+R I s)}\right)}{a R}-I s$

$>I R:=t \rightarrow A(t)$;

$I R:=t \rightarrow A(t)$

$>V A B:=t \rightarrow R \cdot I R(t)$

$V A B:=t \rightarrow R I R(t)$

$>V B C:=t \rightarrow V(t)-V A B(t)$

$V B C:=t \rightarrow V(t)-V A B(t)$

The numerical values used are such that: 
$R:=10: T:=300: I s:=10^{\wedge}(-9): q:=1.60219 * 10^{* *(-19): k B}$

$:=1.38062 * 10 * *(-23):$ eta $:=1.8: \mathrm{Vm}:=400: P:=0.01$ :

$>I R(t) ; \operatorname{VAB}(t) ; \operatorname{VBC}(t) ; R d(t):=\frac{1}{((I s+I R(t)) \cdot a)} ;$

$0.004653223400 \quad$ LambertW $\quad\left(2.149047905 \quad 10^{-7}\right.$

$\mathrm{e}^{8596.191620 t-42.98095789)-} \frac{1}{1000000000}$

0.04653223400 LambertW(2.149047905 $10^{-7} e^{8596.191620 t-}$

$42.98095789)-\frac{1}{100000000}$

400t-1.999999990-0.04653223400

LambertW $\left(2.149047905^{-7} \mathrm{e}^{8596.191620 t-4298095789}\right)$

$R d:=t \rightarrow \frac{1}{(I s+I R(t)) a}$

The following figures are obtaining.

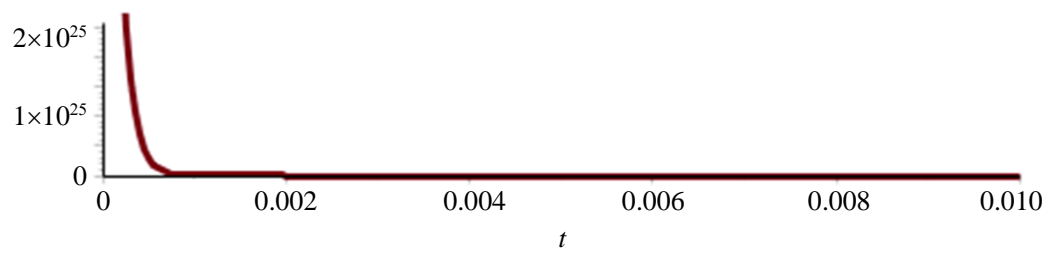

Fig. 8: Graphical representation of the dynamic resistance of the diode in the interval of time $[0, P]$

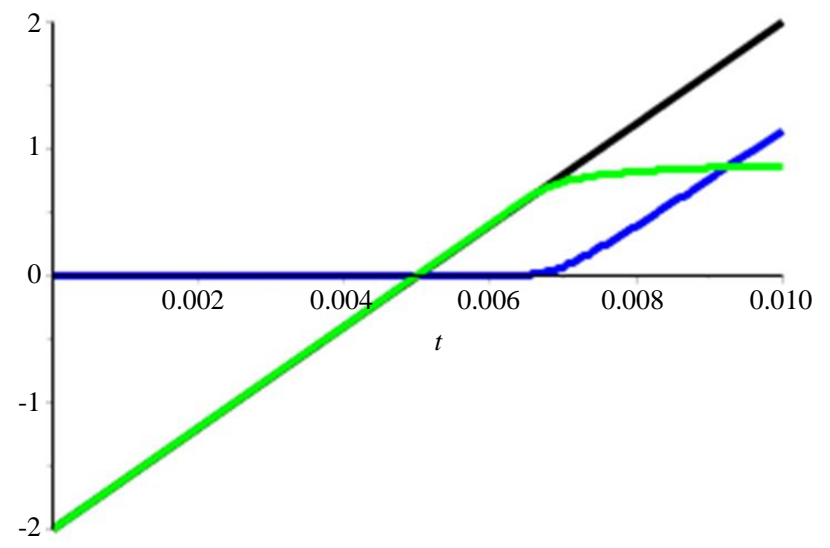

Fig. 9: Graphical representation of voltages at the terminals of the generator, the resistance and the diode in the interval of time [0;0,01]

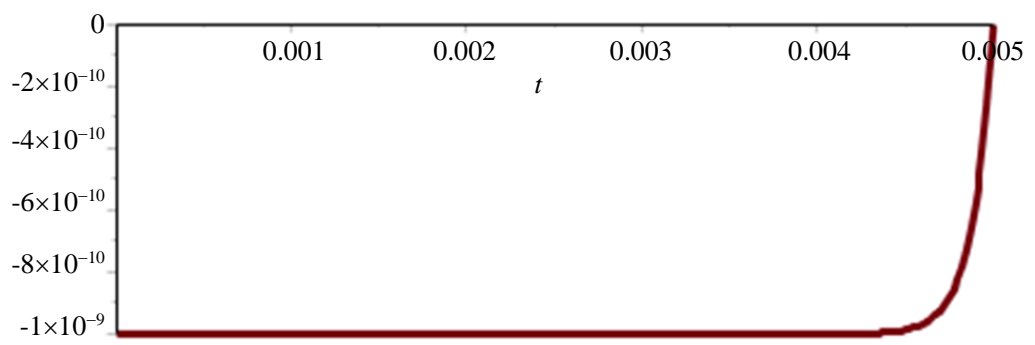

Fig. 10: Graphical representation of current flowing through the resistance over time $[0, P / 2]$ 


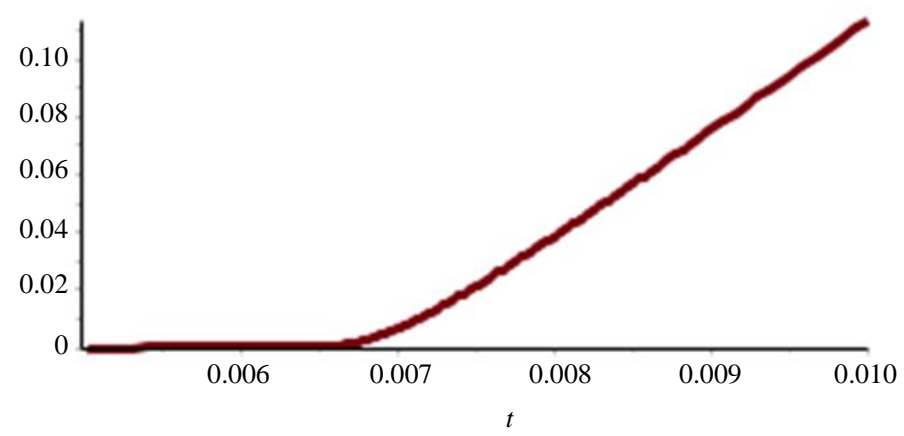

Fig. 11: Graphical representation of current flowing through resistance over time $[\mathrm{P} / 2, \mathrm{P}]$

Graphical Representation of Current Flowing through Resistance Over Time [P/2, P].

$>\operatorname{plot}\left(I R(t), t=\frac{P}{2} . . P\right)$

\section{Influence of the Loading Resistance, Saturation Current, Temperature and Ideality Factor:}

\section{Influence of the Loading Resistance}

Here, we present the influence of the loading resistance on $\mathrm{V}(\mathrm{t}) ; \mathrm{VAB}(\mathrm{t})$ and $\mathrm{VBC}(\mathrm{t})$.

$>$ restart:

$>$ with(plots):

$>V:=t \rightarrow V m^{*}\left(t-\frac{P}{2}\right): a:=\frac{q}{\text { eta.kB.T}}:$

$>\mathrm{A}:=t \rightarrow\left(\frac{1}{a^{*} R}\right) *\left(\operatorname{LambertW}\left(a^{*} R^{*} I s^{*} \exp \left(a^{*}(V(t)+\right.\right.\right.$ $\left.\left.\left.R^{*}(s)\right)\right)\right)-I s$

$>I R:=t \rightarrow A(t)$

$>V A B:=t \rightarrow R \cdot I R(t)$ :

$>V B C:=t \rightarrow V(t)-V A B(t)$ :

$>R d(t):=\frac{1}{(I s+I R(t)) \cdot a}:$

$>T:=300: q:=1.60219 * 10 * *(-19):$ Is $:=10 \wedge(-9): k B:=$ $1.38062 * 10 * *(-23)$ :

$>$ eta $:=1.8: V m:=400: P:=0.01$ :

In the following figure, we present an animation of the voltages $V(t), V A B(t), V B C(t)$ for $\mathrm{R}=0.1 . .100$

>\#animate $(\{V(t), V A B(t), V B C(t))\}, t=0 . .0 .017$, $R=0.1 . .100$;

$>$ animate $(V(t), t=0 . . P, R=0.1 . .100$, color $=$ red $): F:=\%$ :

$>$ animate $(V A B(t), t=0 . . P, R=0.1 . .100$, color $=$ green $)$ : $G:=\%$ :

$>$ animate $(\operatorname{VBC}(t), t=0 . . P, R=0.1 . .100$, color $=$ blue $)$ : $H:=\%$ :
$>\operatorname{display}(\{F, G, H\})$;

Influence of the Loading Resistance on the IR

$>\operatorname{animate}(\operatorname{IR}(t), t=0 . . P, R=0.1 . .100$, color $=B L A C K)$

Influence of the Loading Resistance on the Rd

$>\operatorname{animate}(R d(t), t=0 . . P, R=0.1 . .100$, color $=B L A C K)$

\section{Influence of the Saturation Current}

> restart: with(plots):

$>V:=t \rightarrow V m^{*}\left(t-\frac{P}{2}\right): a:=\frac{q}{\text { eta.kB.T}}:$

$>A:=t \rightarrow\left(\frac{1}{a^{*} R}\right) *\left(\operatorname{LambertW}\left(a^{*} R^{*} I s^{*} \exp \left(a^{*}(V(t)+\right.\right.\right.$ $R *(s))))-I s$ :

$>I R:=t \rightarrow A(t)$ :

$>V A B:=t \rightarrow R \cdot I R(t)$

$>V B C:=t \rightarrow V(t)-V A B(t)$ :

$>\operatorname{Rd}(\mathrm{t}):=\frac{1}{(I s+I R(t)) \cdot a}:$

$>R:=8: T:=300: q:=1.60219 * 10 * *(-19)$ :

$>k B:=1.38062 * 10 * *(-23)$ :

$>$ eta $:=1.8: V m:=400: P:=0.01$ :

In the following figure, we present an animation of the voltages $V(t), V A B(t), V B C(t)$ for $I s=10^{\wedge}(-9) . .10^{\wedge}(-1)$

\#animate $\left(\{V(t), V A B(t), V B C(t)\}, t=0 . . P, I s=10^{\wedge}(-\right.$ 9)..10^(-1));

$>$ animate $\left(V(t), t=0 . . P, I s=10^{\wedge}(-9) . .10^{\wedge}(-6)\right.$, color $=$ red): $F:=\%$ :

$>$ animate $\left(\operatorname{VAB}(t), t=0 . . P\right.$, Is $=10^{\wedge}(-9) . .10^{\wedge}(-6)$, color $=$ green): $G:=\%$ :

$>$ animate $\left(V B C(t), t=0 . . P, I s=10^{\wedge}(-9) . .10^{\wedge}(-6)\right.$, color = blue $): H:=\%$ :

$>\operatorname{display}(\{F, G, H\})$; 
M'hamed El Aydi et al. / Journal of Computer Science 2020, 16 (12): 1669.1683 DOI: $10.3844 /$ jessp.2020.1669.1683

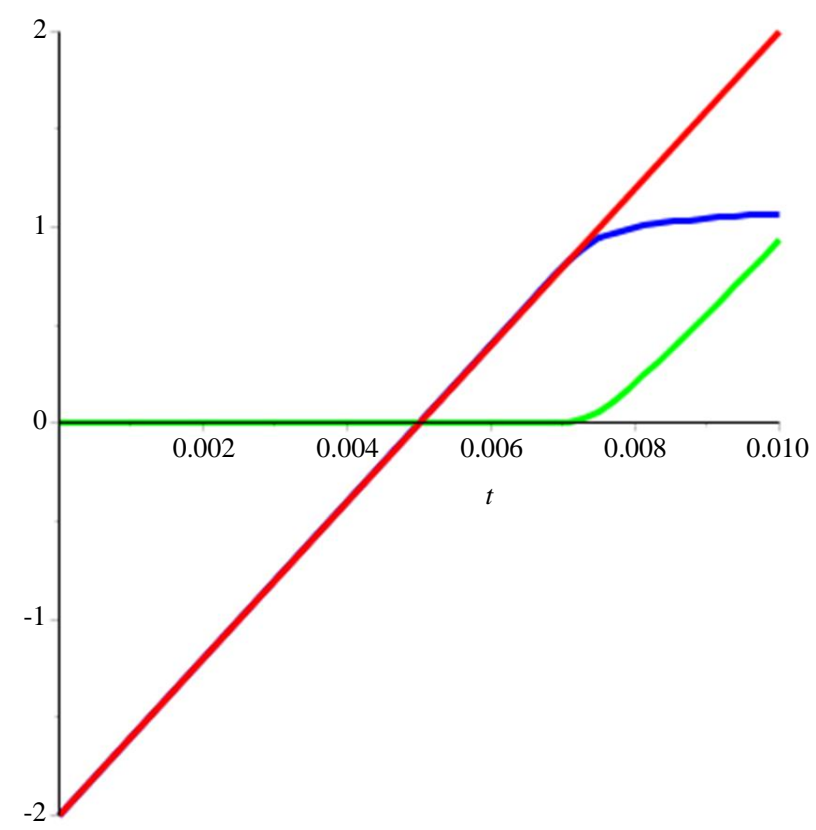

Fig. 12: Influence of the loading resistance on $V(t), V A B(t)$ and $V B C(t)$

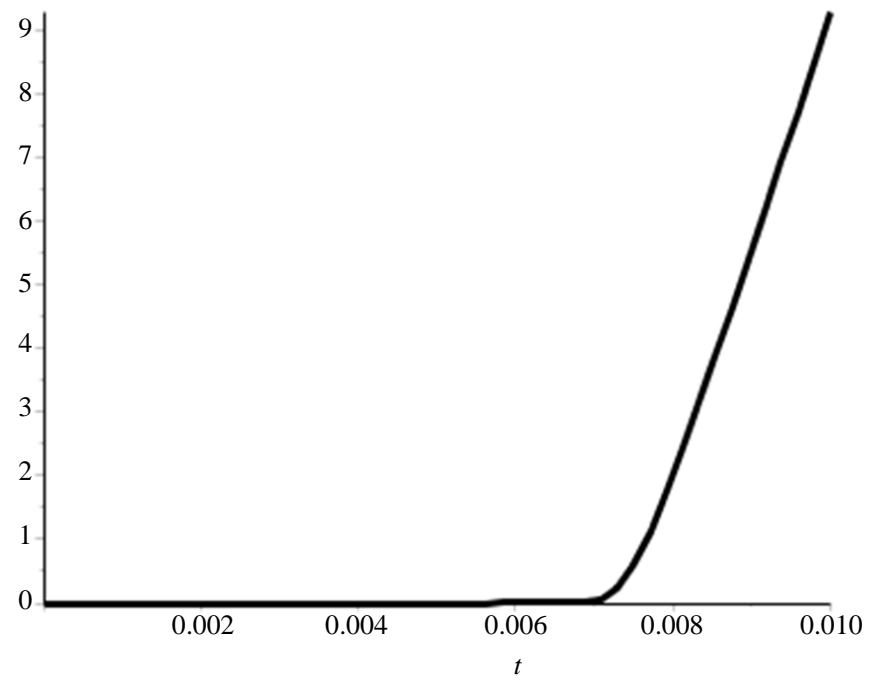

Fig. 13: Influence of the loading resistance on the $I R$

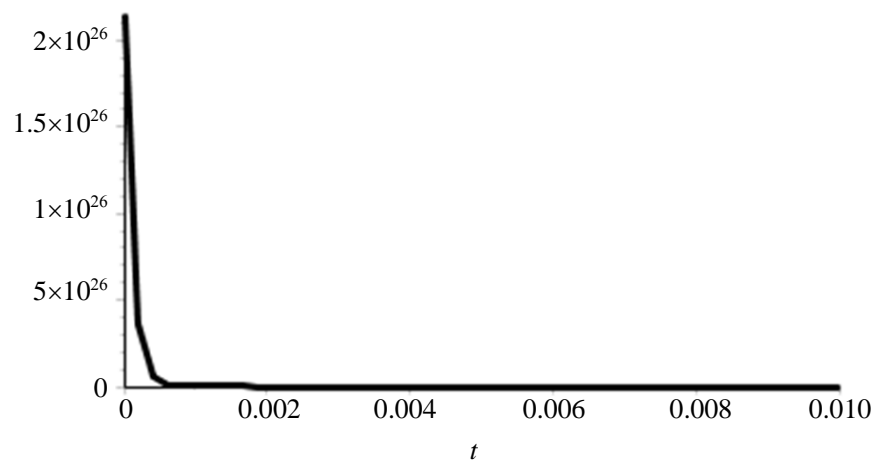

Fig. 14: Influence of the loading resistance on the $R d$ 


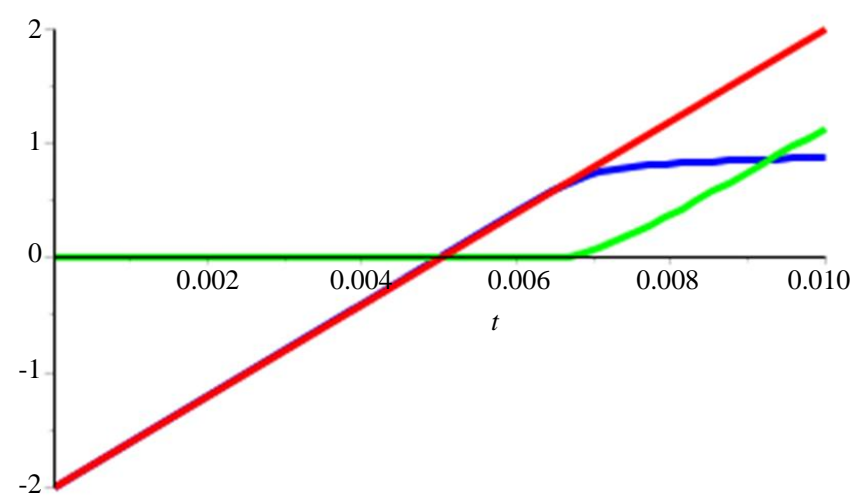

Fig. 15: Influence of the saturation current on $V(t), V A B(t)$ and $V B C(t)$

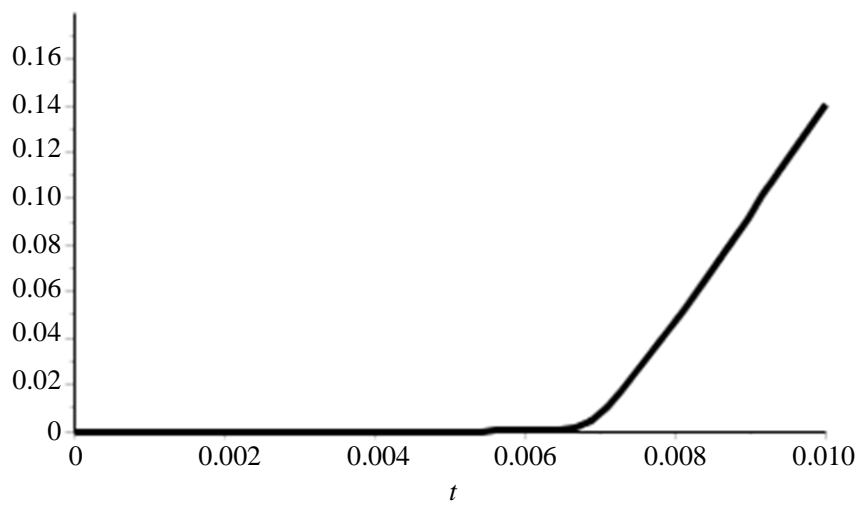

Fig. 16: Influence of the saturation current on the IR

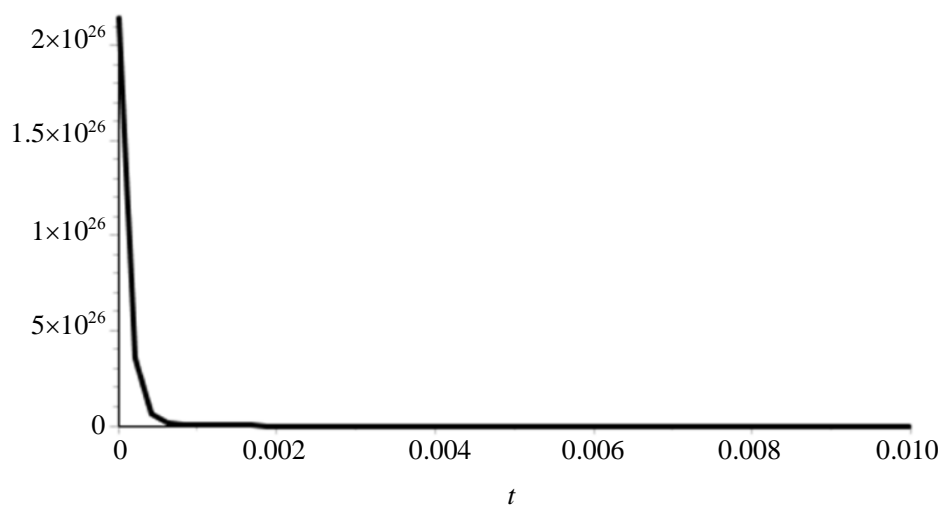

Fig. 17: Influence of the saturation current on the $\mathrm{Rd}$

Influence of the Saturation Current on the IR

$>$ animate $\left(I R(t), t=0 . . P, I s=10^{\wedge}(-9) . .10^{\wedge}(-6)\right.$, color $=$ $B L A C K)$;

\section{Influence of the Saturation Current on the Rd}

$>$ animate $\left(R d(t), t=0 . . P\right.$, Is $=10^{\wedge}(-9) . .10^{\wedge}(-6)$, color $=$ $B L A C K)$

\section{Influence of Temperature}

Influence of Temperature on $V(t) ; V A B(t) ; V B C(t)$

> restart: with(plots):

$>V:=t \rightarrow V m^{*}\left(t-\frac{P}{2}\right): a:=\frac{q}{\text { eta.kB.T}}:$ 


$$
\begin{aligned}
& A:=t \rightarrow\left(\frac{1}{a^{*} R}\right) *\left(\operatorname { L a m b e r t W } \left(a ^ { * } R ^ { * } I s ^ { * } \operatorname { e x p } \left(a ^ { * } \left(V(t)+\quad>R d(t):=\frac{1}{(I s+I R(t)) \cdot a}:\right.\right.\right.\right. \\
& R * I s))))-I s: \\
& >R:=10: q:=1.60219 * 10 * *(-19) \text { : } \\
& >I R:=t \rightarrow A(t) \\
& >V A B:=t \rightarrow R \cdot I R(t) \\
& >I s:=10^{\wedge}(-9): k B:=1.38062 * 10^{* *}(-23) \text { : } \\
& >\text { eta }:=1.8: \mathrm{Vm}:=400 ; P:=0.01 \text { : }
\end{aligned}
$$

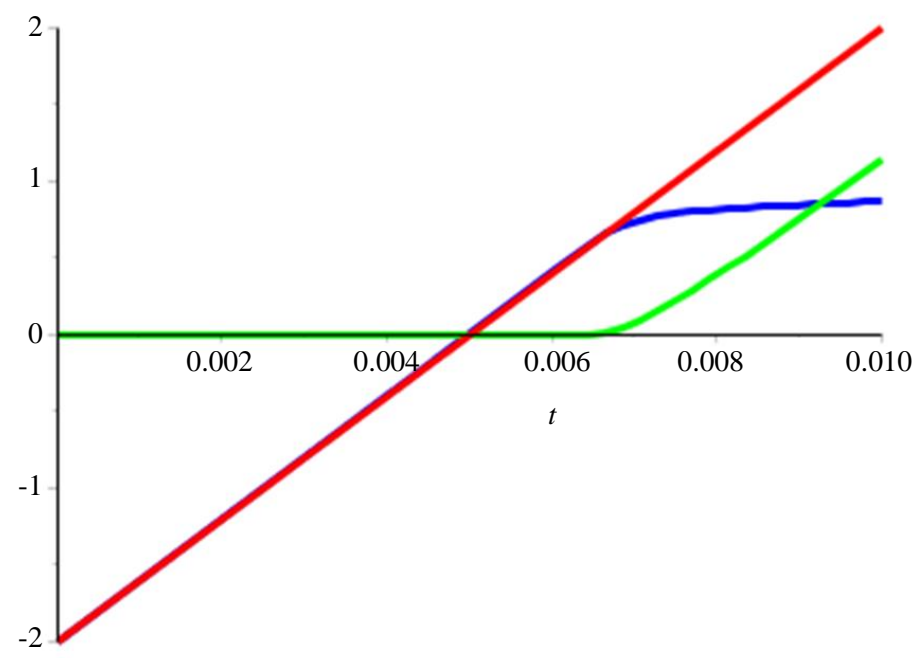

Fig. 18 : Influence of temperature on $V(t) ; V A B(t) ; V B C$

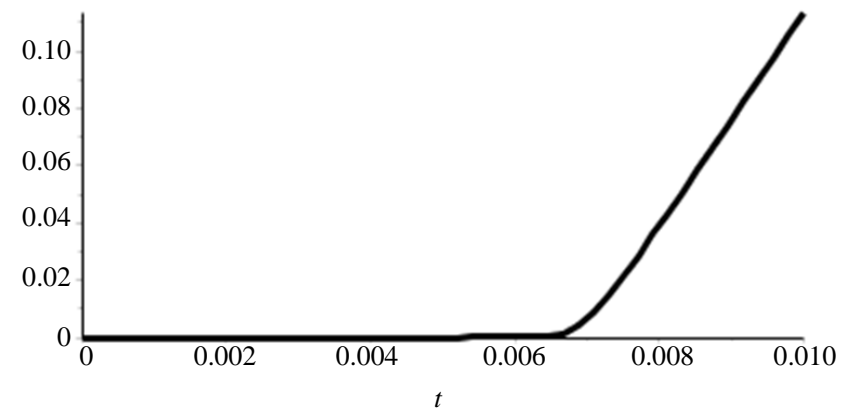

Fig. 19: Influence of the temperature $T$ on the IR current

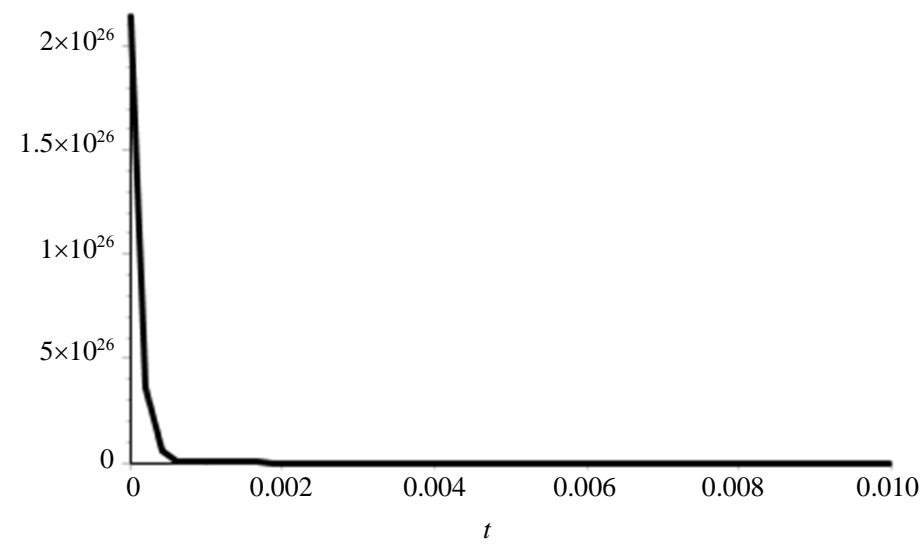

Fig. 20: Influence of the temperature $T$ on the dynamic resistance 
In the following figure, we present an animation of the voltages $V(t), V A B(t), V B C(t)$ for $T=300 . .400)$

$>$ \#animate $(\{V(t), V A B(t), V B C(t))\}, t=0 . . P, T=$ 300..400);

$>$ animate $(V(t), t=0 . . P, T=300 . .400$, color $=$ red $): F:=\%$ :

$>$ animate $(\operatorname{VAB}(t), t=0 . . P, T=300 . .400$, color $=$ green $)$ :

$G:=\%$

$>$ animate $(\operatorname{VBC}(t), t=0 . . P, T=300 . .400$, color $=$ blue $)$ :

$H:=\%$ :

$>\operatorname{display}(\{E, G, H\})$;

Influence of the Temperature T on the IR Current

$>$ animate $(\operatorname{IR}(t), t=0 . . P, T=300 . .400$, color $=B L A C K)$;

Influence of the Temperature $T$ on the Dynamic Resistance

$>\operatorname{animate}(R d(t), t=0 . . P, T=300 . .400$, color $=B L A C K)$

\section{Influence of Ideality Factor}

Influence of Ideality Factor on $V(t) ; V A B(t)$ and $\operatorname{VBC}(t)$

$>$ restart:

$>$ with(plots):

$>V:=t \rightarrow V m^{*}\left(t-\frac{P}{2}\right): a:=\frac{q}{\text { eta.kB.T}}:$

$>A:=t \rightarrow\left(\frac{1}{a^{*} R}\right) *\left(\operatorname{LambertW}\left(a^{*} R^{*} I s^{*} \exp \left(a^{*}(V(t)+\right.\right.\right.$

$\left.\left.\left.R^{*}(s)\right)\right)\right)-I s$ :

$>I R:=t \rightarrow A(t)$ :

$>V A B:=t \rightarrow R \cdot I R(t)$ :

$>V B C:=t \rightarrow V(t)-V A B(t)$ :

$>R d(t):=\frac{1}{(I s+I R(t)) \cdot a}:$

$>R:=8: T:=300: q:=1.60219 * 10 * *(-19): k B:=$ $1.38062 * 10 * *(-23)$ :

$>I s:=10^{\wedge}(-9): V m:=400: P:=0.01$ :

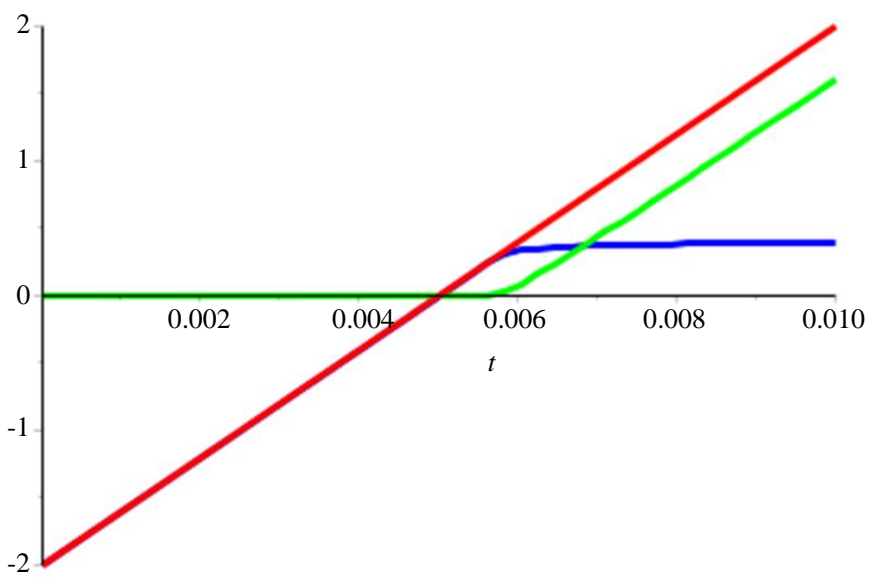

Fig. 21: Animation of $V(t) ; V A B(t)$ and $V B C(t)$ for $t$ in $[0, P]$ and the ideality factor varies between 0.8 and 2

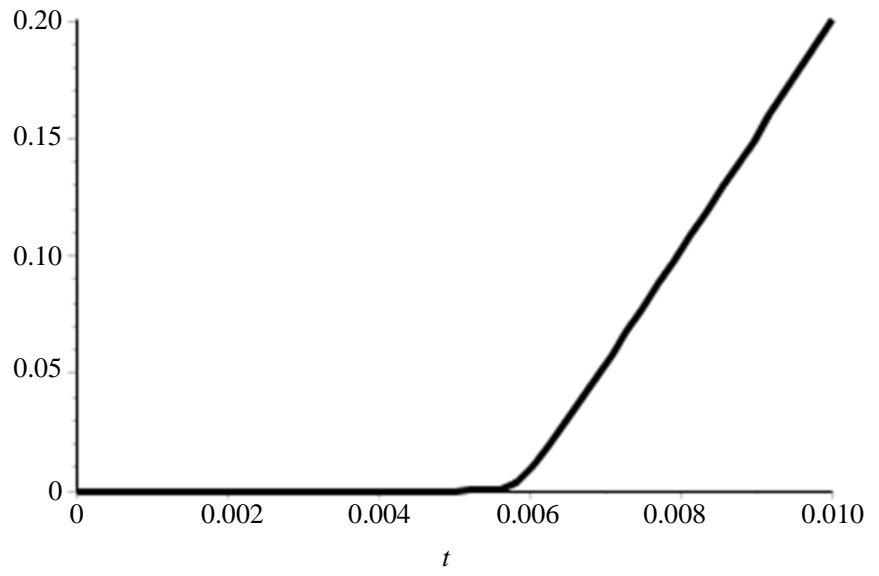

Fig. 22: Animation of $I R(t)$ for $t$ in $[0, P]$ and the ideality factor varies between 0.8 and 2 


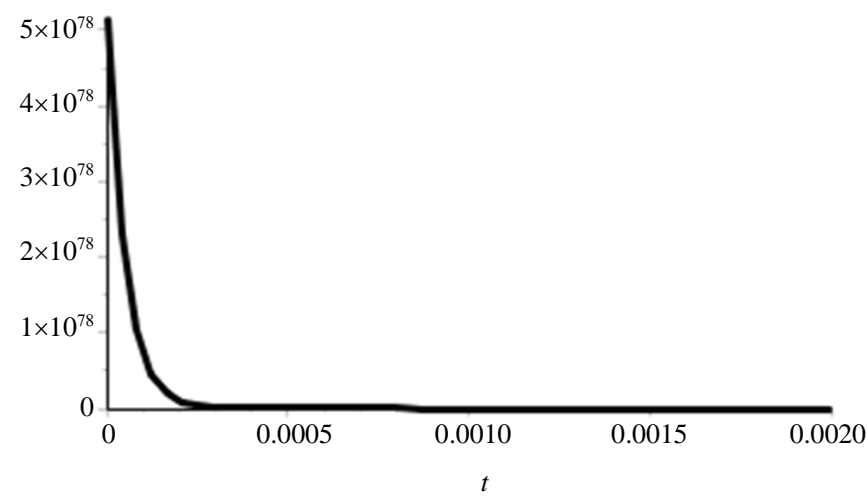

Fig. 23: Animation the dynamic resistance for $t$ in $[0,0.002]$ and the ideality factor varies between 0.8 and 2

Table 1: The influence of load resistance, saturation current, temperature and ideality factor

\begin{tabular}{lllll}
\hline When & $R$ : Increases & $I_{s}:$ Increases & $T:$ Increases & $\eta:$ Increases \\
\hline VAB & Grow & Grow & Decreases & Decreases \\
VBC & Decreases & Decreases & Grow & Grow \\
IR & Decreases & Grow & Decreases & Decreases \\
$R_{d}$ & Invariable & Decreases & Decreases & Decreases \\
\hline
\end{tabular}

In the following figure, we present an animation of the voltages $V(t), V A B(t), V B C(t)$ for $e t a=0.8 . .2$.

$>$ \#animate $(\{V(t), V A B(t), V B C(t)\}, t=0 . . \mathrm{P}$, eta $=0.8 . .2)$;

$>$ animate $(V(t), t=0 . . P$, eta $=0.8 . .2$, color $=r e d): F:=\%:$;

$>$ animate $(V A B(t), t=0 . . P$, eta $=0.8 . .2$, color $=$ green $)$ : $G:=\%$ :

$>$ animate $(\operatorname{VBC}(t), t=0 . . \mathrm{P}$, eta $=0.8 . .2$, color $=$ blue $): \mathrm{H}$ $:=\%$ :

$>\operatorname{display}(\{F, G, H\})$;

\section{Influence of: Ideality Factor on IR(t)}

$>\operatorname{animate}(\operatorname{IR}(t), t=0 . . P$, eta $=0.8 . .2$, color $=B L A C K)$;

\section{Influence of Ideality Factor on Dynamic Resistance}

$>\operatorname{animate}(R d(t), t=0 . . P$, eta $=0.8 . .2$, color $=B L A C K)$;

and the ideality factor varies between 0.8 and 2 .

\section{Results Discussion of the First Problem}

- The current flowing through the resistor:

$$
\begin{aligned}
& I R \quad:=\quad t \rightarrow \quad \frac{1}{a \cdot R} \cdot \operatorname{LambertW}(a \cdot R \cdot(I s 1+ \\
& I s 2) \cdot \exp (a \cdot(R \cdot \cdot(I s 1+I s 2)+V(t))))-(I s 1+I s 2)
\end{aligned}
$$

- Voltage across the resistance $R$ :

$V A B:=t \rightarrow R \cdot I R(t) ;$

- Voltage across the diodes:

$V B C:=t \rightarrow V(t)-R \cdot I R(t) ;$
- $\quad$ The current flowing through the diodes $D 1$ and $D 2$ :

$$
\begin{aligned}
& I 1:=t \rightarrow I s 1 \cdot(\exp (a \cdot V B C(t))-1) \\
& I 2:=t \rightarrow I s 2 \cdot(\exp (a \cdot V B C(t))-1)
\end{aligned}
$$

- $\quad$ Dynamical resistance of the diodes $D 1$ and $D 2$ :

$$
\begin{aligned}
& R d 1:=\frac{1}{(I s 1-I 1(t)) \cdot a} \\
& R d 2:=\frac{1}{(I s 2-I 2(t)) \cdot a}
\end{aligned}
$$

Graphs of current flows and voltages in the circuit as a function of time:

Figure 3 shows that:

- Voltage $V A B$ is positive on $[0, P / 2]$, zero on $[P / 2, P]$ and it reaches a maximum for $t=P / 4$

- Voltage $V B C$ is positive on $[0, P / 2]$, negative on $[P / 2, P]$ and it reaches a minimum for $t=3 P / 4$

Figure 4 shows that:

- the intensities of the currents $I R, I 1$ and $I 2$ are positive on $[0, P / 2]$ and they reach their maximums at the same time $t=P / 4$

- the intensities of the $I R$ currents, $I 1$ and $I 2$ are zero on $[P / 2, P]$, this is the role played by the diodes

Figure 5 shows that:

- $\quad$ The intensities of the current $I 1$ and $I 2$ are negative and almost null $[P / 2, P]$, because the diodes are not ideal

Figure 6 shows that: 
- The dynamic resistances $R d 1$ and $R d 2$ are almost zero on $[0, P]$ except on a small interval of time

- Over this small time interval, we notice that $R d 1$ and $R d 2$ are positive and they reach their maximums at the same time

- $\quad R d 2$ is greater than $R d 1$ because $I s 2$ is less than $I s 1$

\section{Results Discussion of the Second Problem}

- The current flowing through the resistor IR:

$$
\begin{aligned}
& A:=t \rightarrow\left(\frac{1}{a^{*} R}\right) *\left(\text { Lambert } \mathrm { W } \left(a ^ { * } R ^ { * } I s ^ { * } \operatorname { e x p } \left(a^{*}(V(t)+\right.\right.\right. \\
& \left.\left.\left.\left.R^{*} I s\right)\right)\right)\right)-I s \\
& I R:=t \rightarrow A(t)
\end{aligned}
$$

- Voltage across the resistance R:

$\mathrm{VAB}:=t \rightarrow R \cdot I R(t)$

- Voltage across the diode:

$V B C:=t \rightarrow V(t)-R \cdot I R(t) ;$

- Dynamical resistance of the diode D:

- $\quad R d(t):=\frac{1}{(I s 1-I R(t)) \cdot a}$ :

The graphical representations allowed us to manipulate commands of the Maple software and to deduce the following results:

Figure 8 shows that:

- The dynamic resistance of the diode decreases very quickly in the time interval] $0,0.001]$ then it remains zero

Figure 9 shows:

- The voltage across the generator is a straight segment

- For $0<t<t_{0},\left(0.006<t_{0}<0.007\right)$, the voltage $V B C$ coincides with $V$ and that $V A B$ is zero

Figure 10 and 11 shows:

- For $0<t<P / 2$, the IR current in the resistor is zero. the current $I R$ increases and $i$ is positive for $P / 2<t<P$

The influence of load resistance, saturation current, temperature and ideality factor:

- The results of Fig. 12 to 23 are summarized in the following Table 1

\section{General Discussion}

The use of the Maple software allowed us to solve transcendent equations, to analytically express the solutions according to the LAMBERT $\mathrm{W}$ functions, to represent the solutions graphically and to make animations.

Important question: Why don't we teach the LAMBERT $\mathrm{W}$ function and the Maple software at the secondary school?

\section{Conclusion}

We used the Maple software in several directions:

- Modeling and problem solving, determination of exact analytical solutions of the expressions of current and voltage in the electronic circuit, Visualization of their evolutions as a function of time and illustration of the influence of certain parameters on the current and the voltages

- This work is of interest to researchers in mathematics, physicists and teachers of mathematics or physics

- The user of this article can make the following changes:

i) Change the amplitude value $V m$ sinusoidal signal and watch the changes in graphs and animations

ii) Change the physical parameters for example the temperature and look for the effect produced on the graphs and the animations

iii) Substitute one signal for another and look at the influence on graphs and animations

\section{Author's Contributions}

All authors equally contributed in this work.

\section{Conflicts of Interest}

The author declares no conflicts of interest regarding the publication of this paper.

\section{Ethics}

This article is original and contains unpublished material. The corresponding author confirms that all of the other authors have read and approved the manuscript and no ethical issues involved.

\section{References}

Banwell, T. C., \& Jayakumar, A. (2000). Exact analytical solution for current flow through diode with series resistance. Electronics letters, 36(4), 291-292.

Dence, T. P. (2013). A brief look into the Lambert W function.

Eberhart, C. (2009). Maple Problem Solving Handbook. 
Fjeldly, T. A., Moon, B. J., \& Shur, M. (1991). Approximate analytical solution of generalized diode equation. IEEE transactions on electron devices, 38(8), 1976-1977.

Pimbley, J. M., Fjeldly, T. A., Moon, B., \& Shur, M. (1992). Iterative solutions of the generalized diode equation. IEEE transactions on electron devices, 39(5), 1268-1269.
Vargas-Drechsler, M. A. (2005). Analytical Solution of Diode Circuits. Maple application center. 